Página inicial: 167 - Página final: 182

TIPO DE ARTíCULO: de investigación

\title{
LAS METAS DEL MILENIO Y LOS PRINCIPIOS DEL COMERCIO JUSTO
}

\author{
THE MILLENNIUM GOALS AND THE PRINCIPLES OF FAIR TRADE
}

Recibido: Octubre de 2011 - Revisado: Noviembre de 2011 - Aceptado: 30 de Noviembre de 2012

Por: Marco Coscione ${ }^{1}$

\section{RESUMEN}

El presente articulo presenta el resultado de una investigación que busca sistematizar y visibilizar la forma como los pequeños productores trabajan para el desarrollo humano, sostenible y el cumplimiento de las metas del Milenio en República Dominicana

\section{PALABRAS CLAVE:}

desarrollo humano, desarrollo sostenible, metas del milenio, campesinos

\section{ABSTRACT:}

This paper presents the results of an investigation whose aim is to systematize and to make visible the way that small producers work for sustainable human development and goal attainment of the Millennium in the Dominican Republic.

\section{KEY WORDS:}

Human Development, Sustainable Development, the Millennium Goals, Peasants.

\footnotetext{
${ }^{1}$ Licenciado en Ciencias Internacionales y Diplomáticas por la Universidad de Génova, Diplomado sobre "E1 Futuro de la Unión Europea y sus relaciones con América Latina. Hacia Viena 2006” (Universidad de Chile) y el Máster Oficial en "América Latina Contemporánea y sus relaciones con la UE: una cooperación estratégica" (Universidad de Alcalá e Instituto Universitario de Investigación Ortega y Gasset, Madrid). Tiene varias experiencias de estudio, trabajo y voluntariado en Europa (Italia, Alemania y España) y en América Latina (Cuba, Chile, Perú, El Salvador, Rep. Dominicana), Actualmente labora en República Dominicana. marco.coscione@gmail.com
} 


\section{Introducción}

Es opinión de muchos economistas, politólogo, expertos en relaciones internacionales y no solamente, que a través del acceso y la participación en el comercio internacional se obtienen resultados muchos más exitosos, por ejemplo en la lucha contra la pobreza y la reducción de las inequidades, que con las ayudas al desarrollo o los programas condicionados de los organismos financieros internacionales o de las agencias de cooperación. Sin embargo, en el comercio internacional siguen existiendo trabas y barreras que a unos impiden las posibilidades de desarrollo y a otros garantizan los niveles de bienestar adquiridos con el tiempo, con la explotación y especulaciones. El llamado "mercado libre", en realidad es libre solo para pocos grandes, los mismos que ponen las condiciones y escriben las reglas del juego. Sin embargo, el mero acceso a los mercados globales no garantiza reducciones en los índices de pobreza o desigualdad, ni tampoco es sinónimo de un verdadero desarrollo económico, social y político. No es casualidad, de hecho, que la enorme expansión que ha conocido el comercio mundial en las últimas décadas no haya reducido las desigualdades y las injusticias entre los países del Norte y los del Sur, sino todo lo contrario: las ha profundizado, dibujando un panorama indefendible moralmente e insostenible bajo todo punto de vista. Las condiciones de partida, ya muy desiguales, se mantuvieron o empeoraron, para que algunos pudiesen seguir conservando o mejorando sus niveles de vida y de riqueza. En la Rep. Dominicana, por ejemplo, a pesar de un crecimiento con tasa promedio del 5.4\% por año en los últimos 50 años, el poder económico, social y político sigue en manos de unos pocos generándose, de este modo, mayor desigualdad y mayor exclusión en todos los niveles, y "convirtiendo al desarrollo humano en una cuestión de poder, cuando debería ser una cuestión de derechos" (PNUD, 2008). Esta fue la principal tesis del Informe de Desarrollo Humano de 2008 del PNUD de República Dominicana, y sigue siendo válida.

En contra de estas injusticias y desigualdades, siempre se han levantado voces de luchas y revolucionarias, se han organizados movimientos ciudadanos nacionales e internacionales. Antes eras "rojos" o "sucios comunistas", ahora son los "no-global", "los anti globalización", aunque en realidad sean todo lo contrario. En la era del capitalismo avanzado y del neoliberalismo, los movimientos sociales se han reorganizado, han cambiado estrategias, han sido cooptados y han vuelto a despertarse, con nuevos objetivos, nuevos actores, nuevas modalidades y respondiendo de distintas formas a la evolución misma del modelo mundial impuesto, proponiendo ahora sí una nueva globalización: la globalización, desde abajo, de los derechos y de la solidaridad. Dentro de estos movimientos globales, el "Movimiento del Comercio Justo" es seguramente uno de los más innovadores y exitosos y, sin duda, uno de los más dinámicos porque no deja de auto cuestionarse, desde adentro y desde abajo, y de conocer "infiltraciones", desde afuera y desde arriba. En poco tiempo, lo que al principio era un "oscuro nicho de mercado" se convirtió en "un fenómeno globalmente reconocido" (Murray \& Raynolds, 2007).

\section{Metodologia}

Para el desarrollo de este trabajo no se retomará tanto la historia del Comercio Justo (en adelante CJ) o sus actuales desafios, sino más bien se subrayarán la importancia del enfoque multidimensional o multisectorial que se implementa a través de relaciones comerciales justas y solidarias. Se hará comparando los principios fundamentales del CJ con las Metas del Milenio, posteriores de varias décadas al nacimiento del movimiento, y cuyo alcance para el 2015 solo en mínima parte será posible. Y se hará relacionando ejemplos muy concretos de 
cómo las organizaciones de pequeños productores de comercio justo, presentes en el país (República Dominicana) en los rubros de café, cacao y banano, trabajan cotidianamente para el desarrollo humano y sostenible de sus miembros, de las respectivas familias y comunidades. Entonces de todo el país.

Las organizaciones consideradas en esta investigación son 11:

\begin{tabular}{|c|c|c|}
\hline Café & Cacao & Banano \\
\hline Coscafé & Conacado & Aprobano (Finca 6) \\
\hline Fedecares & Fundopo & Asoarac \\
\hline Junta Monseñor Romero & Junacas & Banelino \\
\hline Coopaprocosemup & & Coopprobata \\
\hline
\end{tabular}

\section{Resultados - discusiones}

\section{Comercio Justo}

Como se decía anteriormente, el mercado no es igualmente libre para todos. Las condiciones de partida influencian el grado de aprovechamiento de sus mecanismos.

Las que algunos prefieren llamar "imperfecciones de mercado" son en realidad verdaderas barreras que impiden a los pequeños y marginalizados productores un buen aprovechamiento de sus propias capacidades productivas y de los canales comerciales. El objetivo de fondo del CJ es la reducción de la pobreza a través de:

La Creación de Oportunidades para los Productores en Desventaja Económica: no se trata de asistencialismo o ayuda al desarrollo. Se trata de cambiar las reglas del juego para que los pequeños productores marginalizados puedan, a través de su trabajo cotidiano, desarrollar sus habilidades, aprovechar las oportunidades del mercado y entonces emprender su propio camino hacia el desarrollo. Pero este trabajo tiene que ser digno, cumplir con las leyes nacionales y los convenios internacionales de la Organización Internacional del Trabajo, no contempla el trabajo forzoso y, en la medida de lo posible (y siempre controlado), el trabajo infantil. ${ }^{2}$ Las relaciones de CJ solo se pueden dar si la organización involucrada "proporciona un entorno de trabajo seguro y saludable para los empleados y/o miembros" (World fair trade organization, 2008).

Por trabajo digno se entiende también un sueldo digno. Las organizaciones de CJ pagan el sueldo mínimo o un sueldo por encima del sueldo mínimo nacional, sin discriminaciones: "Los salarios que pagamos nosotros", me contaba Simeón Ramírez de la de la Asociación de Agricultores Ramón Antonio Cruz (Asoarac), "están por encima del salario mínimo y de lo que se suele pagar en esta zona. Aquí el promedio de un peón son 200 pesos diarios, y con diferencias entre haitianos y dominicanos; nosotros pagamos 300 a todos, además, damos desayuno y comida, cuando podemos transporte, y garantizamos seguro social". ${ }^{3}$ En un país como Rep. Dominicana, donde por lo menos el $25 \%$ de la población recibe menos del salario mínimo y donde los diferenciales salariales entre hombres y mujeres, nacionales e haitianos son aún muy altos, las organizaciones de CJ reman contra la corriente. 
A continuación, se analizan las principales barreras que impiden este aprovechamiento, y entonces las respuestas que a ellas proporcionan las relaciones de CJ. Este primer análisis nos ayudará a entender los principios básicos del movimiento y cómo el CJ participa directamente en la lucha contra la pobreza, el hambre y la desigualdad.

Falta de acceso al mercado: los pequeños productores se encuentran en zonas de no fácil acceso, no producen volúmenes suficientes, no disponen de transportes adecuados y están obligados a vender los productos a los intermediarios.

El CJ ofrece Acceso Directo al Mercado; las organizaciones de productores rompen con el yugo de los intermediarios, reconocido también por todas las organizaciones dominicanas como uno de los principales problemas que tienen los pequeños productores para progresar. "Ahora somos nosotros que le hacemos competencia a los intermediarios", bromeaba Corporino Félix, uno de los pequeños productores más representativo de la Cooperativa Agropecuaria de Productores de Café Orgánico y Servicios Múltiples de Polo (Barahona). "[...] La cosa más importante, creo yo, es que ya somos nosotros quien decidimos cómo funciona el mercado del café en esta zona. Ahora, el intermediario local espera saber a cuanto Coscafé comprará el café a sus socios para fijar el precio que él pagará a los demás", decía José Antonio Rosario, actual presidente de la Junta Directiva de Coscafé.

Altibajos en los mercados internacionales: los pequeños productores están completamente expuestos a las fluctuaciones de los precios, que les impiden planear la producción y la comercialización a largo plazo.

El Precio Mínimo Garantido, que en teoría debería igualar los costos de producción, los costos para llevar una vida digna y los costos para cumplir con los estándares del CJ, pero que en la práctica tiene que también tiene que mediar con las leyes de la oferta y de la demanda, garantiza seguramente mejores ingresos de los que pudieran garantizar los intermediarios locales. Representa un colchón bajo del cual los integrantes del movimiento (tanto productores como importadores y consumidores) no pueden negociar. Sin embargo, cuando el precio de bolsa es mayor del mínimo de CJ, las transacciones comerciales "justas" también se desarrollan a partir del precio en los mercados internacionales. Además de garantizar un precio mínimo, las organizaciones de CJ establecen Relaciones Comerciales de Largo Plazo.

Todos los miembros de las organizaciones recuerdan que el elemento central de estas nuevas relaciones es la estabilidad y la visión de futuro: poder contar con un pedido seguro durante varios años a veces es más importante que un precio más justo. Coopprobata vende casi la totalidad de su exportación a la comercializadora europea AGROFAIR, de la cual también es socia; Yoel Tejeda, presidente de la cooperativa, me comentaba que la relación con AGROFAIR es muy buena, y que "ser socio tiene grandes beneficios que no dependen solo del precio justo o de los dividendos, sino sobre todo de las relaciones estables, solidarias y a largo plazo que se mantienen al interior de AGROFAIR".

Falta de información o informaciones imperfectas: los pequeños productores no tienen los medios suficientes para poder cotidianamente recibir informaciones sobre los mercados y los productos; esta carencia, por ejemplo, hace imposible conservar temporáneamente algunos productos para venderlos posteriormente a precios más favorables y aumenta la dependencia hacia los intermediarios que pueden tener acceso a todo tipo de información. 
Las organizaciones de CJ del Norte proporcionan informaciones sobre los mercados, los precios, la calidad de los productos o los requisitos de los mercados del Norte. Todas estas informaciones son compartidas y no son monopolios de los intermediarios o de las multinacionales de turno. El desequilibrio informativo se corrige, porque las Relaciones Comerciales deben de ser Transparentes, de Confianza y de Largo Plazo y contemplar una constante Rendición de cuentas, tanto hacia el consumidor, como entre los mismos miembros asociados. Algo que a las mismas autoridades del Estado se les olvida diariamente. Falta de acceso al crédito: los pequeños productores dificilmente acceden a los bancos convencionales, y si les conceden un crédito lo hacen a tasas muy elevadas. Los bancos rurales o comunitarios, que pudieran dar respuesta a las particulares exigencias de los pequeños productores, no están todavía desarrollados como para responder a estas necesidades.

Elemento clave en las relaciones de CJ es el Prefinanciamiento, o pago adelantado, por parte de las importadoras "justas", de por lo menos un 50\% del valor del producto final. Sin embargo, no siempre las organizaciones del Norte cumplen con el principio del prefinanciamiento, y las organizaciones de productores tampoco lo solicitan. El problema es entonces doble: las organizaciones del Norte esperan que el productor lo reclame y el productor no realiza los trámites requeridos. FEDECARES tiene compradores que pagan hasta un $60 \%$, pero para la organización es más importante garantizar el producto; eso quiere decir convencer a los productores a que sigan vendiendo su café a la Federación, aunque en el corto plazo algún intermediario pueda ofrecer un mejor precio: "De esta manera el productor podría llenarse el bolsillo un día pero se le olvidaría su futuro y su estabilidad", afirmaba Manuel Andujar, actual Secretario General de FEDECARES y miembro del Movimiento Cafetalero de Acción Comunitaria (MOVICAC). "Si el mercado no estuviese en estas buenas condiciones, el prefinanciamiento sería perfecto. Pero con buenos precios en los mercados internacionales, nos arriesgamos a que nosotros recibamos el pago adelantado y después el productor venda al intermediario".

Imposibilidad de cambiar ágilmente tipo de producción: además de tener serios problemas técnicos para generar el cambio, los pequeños productores muy raramente consideran la posibilidad de arriesgarse con otro cultivo, sin tener la seguridad de que su situación (muy pobre pero que les permite sobrevivir) mejorará.

Es un elemento al cual las organizaciones de CJ (no tanto las importadoras, sino sobre todo las productoras) responden a través de la Diversificación de la producción: todas las organizaciones de productores dominicanos de CJ la implementan no solamente para vender diferentes productos, sino también para sostener el consumo familiar (entonces diferencias las aportaciones alimenticias a unas dietas muy poco variadas) y alimentar los suelos (el caso de las fincas ecológicas en biodiversidad de Banelino es seguramente el más emblemático). Sin embargo, vale la pena recordar el caso de Junacas, pequeñas organización de Salcedo cuyos productores (en la actualidad solo 72) han logrado convertirse en cacaocultores dejando progresivamente, pero de manera efectiva, su anterior producción cafetalera. En este caso, "la crisis del sector cafetalero obligó los productores a cambiar de rumbo", recuerda Felipe Díaz, "pero sin unir nuestros esfuerzos para la comercialización colectiva del producto no lo hubiéramos logrado". Es gracias a este espíritu que Junacas, hace dos años, pudo entrar en los circuitos del CJ. 
Debilidades organizativas y comerciales: el pequeño productor tiene escasa formación y en cuanto marginalizado es un sujeto débil. Los mismos problemas de debilidad o de gestión podrían caracterizar la estructura asociativa si ésta no tiene ningún incentivo a mejorar su cohesión interna y la participación de los socios.

El CJ solo trabaja con Organizaciones que mantengan Estructuras Participativas y Democráticas. Todas las organizaciones dominicanas de pequeños productores de comercio justo cumplen escrupulosamente con este principio, y de la unión hacen su fuerza: en todas, la Asamblea general de los productores socios es la que toma las decisiones a largo plazo, traza las líneas directrices del plan de desarrollo interno y se reúne extraordinariamente todas las veces que se necesite. Las Juntas Directivas están llamadas a ejecutar los mandatos de la Asamblea y de tomar las necesarias decisiones de corto plazo. "FEDECARES tiene una estructura totalmente democrática", me decía María Isabel Balbuena. "Cualquier socio puede ser elegido y puede participar en las asambleas. Se hacen asambleas ordinarias mensualmente, de Junta y de delegados, y cada dos años se celebra la asamblea eleccionaria. En la Junta Directiva cada núcleo tiene un representante, estableciendo así una relación directa con cada zona". En las organizaciones de tercer nivel, a nivel de núcleos y de asociaciones se reproduce la misma estructura.

Creación de oportunidades, garantizar un trabajo y un salario dignos, acceso al mercado, precio mínimo garantizado, relaciones de largo plazo, transparentes y de confianza, rendición de cuentas, prefinanciamiento y diversificación de la producción, son todas medidas que influyen directamente o indirectamente en el logro del las diferentes metas del Objetivo de Desarrollo del Milenio número 1: erradicar la pobreza extrema y el hambre. Si consideramos que las organizaciones de pequeños productores, a través de las relaciones de CJ, luchan cotidianamente contra la pobreza y a la desigualdad, tenemos que preguntarnos qué papel jugarian en el futuro del país. Recordemos aquí algunas de las líneas de acción previstas por la Propuesta de Estrategia Nacional de Desarrollo 2010-2030, presentada por el actual Gobierno:

\begin{tabular}{|c|c|}
\hline \multicolumn{2}{|c|}{$\begin{array}{l}\text { EJE 2: Una sociedad cohesionada, con igualdad de oportunidades } \\
\text { y bajos niveles de pobreza y desigualdad }\end{array}$} \\
\hline Objetivo Específico & Líneas de Acción \\
\hline \multirow[t]{2}{*}{$\begin{array}{l}\text { Disminuir y aliviar la pobreza } \\
\text { mediante un efectivo y eficiente } \\
\text { sistema de protección social. }\end{array}$} & $\begin{array}{l}\text { 1) Estimula r y consolidar redes comunitarias que contribuyan al } \\
\text { fortalecimiento del capital social y al abordaje colectivo de los problemas } \\
\text { comunitarios, a fin de mejorar los niveles de convivencia, participación, } \\
\text { condiciones de vida y seguridad ciudadana. }\end{array}$ \\
\hline & $\begin{array}{l}\text { 4) Prom over la participación activa de los diferentes actores y sectores } \\
\text { sociales en los procesos de diseño, ejecución, evaluación y monitoreo } \\
\text { de programas y proyectos orientados a la reducción de la pobreza }\end{array}$ \\
\hline
\end{tabular}

Estas "redes comunitarias" en muchos casos ya existen; hay que empezar a conocerlas, REconocerlas y a proporcionarles recursos adecuados. Si las contribuciones del CJ a la reducción de la pobreza son más evidentes (sobre todo a través de un mejor precio que genera mayores ingresos y una prima social que permite inversiones comunitarias), las consecuencias en la disminución de la desigualdad son más ambivalentes. Por un lado es cierto que, elevando los ingresos de los productores y sus calidades de vida, el CJ está indirectamente disminuyendo el gap entre los más ricos y los más pobres y las desigualdades urbano-rurales, de las cuales el país aún sufre muy claramente; recordamos que el $70 \%$ de la 
población rural recibe el 35\% del ingreso nacional, mientras que el 10\% más rico recibe el $34 \%$. Por el otro lado, no es tan claro cuáles sean las consecuencias al interior de una misma comunidad rural, donde viven y trabajan productores organizados y que exportan al CJ, así como productores que siguen sin tener acceso a este mercado de nicho. Recordamos, por ejemplo, que uno de los criterios del CJ es que "los sueldos deben ser dignos pero no crear graves desigualdades con otros productores locales no ligados al CJ". Sin embargo, de las entrevistas no salen particulares problemas de este tipo. Es más, todos los entrevistados opinan que, a pesar de naturales recelos que pueden haber entre productores de CJ y productores convencionales, no hay riesgo de que estas desigualdades puedan poner en peligro la cohesión y la integridad de una comunidad.

Es por este motivo que las inversiones sociales de la prima de CJ deben necesariamente dirigirse a toda la comunidad, y no solamente a los socios o familiares de los productores asociados. Además, como se subraya en los criterios referentes a las organizaciones de CJ del Norte, éstas "privilegiarán las relaciones con aquellos productores más comprometidos e involucrados en un cambio socioeconómico". Este cambio se refleja claramente en el compromiso comunitario de las organizaciones de productores que participan del CJ.

Aquellas organizaciones que no se involucran en el cambio no pueden ser parte de las relaciones comerciales justas y solidarias promovidas por el movimiento. Este compromiso por el cambio es bien visible en los ámbitos de la educación, de la formación y capacitación, pero también en todos aquellos procesos que generan el crecimiento cualitativo de las organizaciones y el empoderamiento de los sujetos, tanto a través del trabajo como de la participación en la gestión de las organizaciones. La prima o premio de comercio justo (10 céntimos de dólar la libra de café, 200 USD la tonelada de cacao y 1 dólar por caja de $18.14 \mathrm{Kg}$ en el caso del banano) es el instrumento a través del cual las organizaciones de productores garantizan distintas inversiones sociales a favor de sus socios y de las comunidades. Estas inversiones se concentran en los ámbitos claves del desarrollo social, humano y sostenible de las personas y reflejan claramente la multidimensionalidad de la estrategia del Milenio: educación (Objetivo 2: lograr la enseñanza primaria universal), equidad de género (Objetivo 3: promover la igualdad entre los sexos y el empoderamiento de la mujer), salud (Objetivo 4: reducir la mortalidad de los niños menores de 5 años; Objetivo 5: mejorar la salud materna; Objetivo 6: combatir el VIH/SIDA, la malaria y otras enfermedades) y medio ambiente (Objetivo 7: garantizar la sostenibilidad del medio ambiente).

\section{Educación y Empoderamiento}

El país mantiene uno de los niveles más bajo, en el continente latinoamericano y caribeño, de inversión en educación. Entre las recomendaciones finales del Informe "Politica Social: capacidades y Derechos" de la ODH/PNUD (2010: 300 - 310, VOL III), es necesario, aquí, recordar algunas como: "Construcción masiva de escuelas para generalizar la tanda completa e incorporar espacios para comidas y descanso de estudiantes y docentes [...] Dignificar salarialmente las labores de los docentes [...] Desarrollo de los institutos técnicos superiores comunitarios [...] Establecer programas de alfabetización. Creación de programas específicos de formación profesional especializados para grupos específicos, como las mujeres (de bajo nivel educativo o empleadas en sectores de baja productividad)".

La inversión en infraestructuras educativas es una de las prioridades para las organizaciones de productores: justo cuando visité el Bloque2 de CONACADO, por ejemplo, se estaba 
ampliando la escuela de la comunidad de El Cercadillo. El Ministerio de Educación se había comprometido con la finalización de las obras de ampliación, pero al final nunca pudo cumplir. "Así que la comunidad nos pidió que les ayudásemos con la obra", nos explicaba Emilio de la Cruz. Asoarac invirtió recursos propios y de la prima de CJ en la reparación de la Escuela de la comunidad "Doña Antonia" y en la construcción de la verja perimetral de la escuela de Jaibón. Pero también tuvo que asumir los sueldos de un maestro que las autoridades no pudieron (o quisieron) asumir, para que los niños de primero de básica empezaran sus clases. El Festicafé de este año en Polo, en la organización del cual la Cooperativa de Caficultores mantiene un liderazgo importante, fue dedicado "a la niñez de la isla": las componentes culturales y educativas, sobre todo para los más pequeños, estuvieron presentes en todos los talleres de pintura, teatro o danza que las agrupaciones dominicanas, haitianas y de Puerto Rico llevaron a cabo durante el festival. Banelino, organización que invierte en educación el $68 \%$ de todas las inversiones sociales comunitarias, mantiene un interesantísimo proyecto de apoyo directo al "Instituto de Niños Especiales" (IDENE), a través de una escuela especial única en la región, de la cual se benefician 60 niños especiales que provienen de Mao, Palo Verde, Montecristi y otras zonas. Además, patrocina varias escuelas y otras infraestructuras educativas en las diferentes zonas correspondientes a las unidades productivas; entrega útiles escolares, uniformes y becas universitarias, y facilita actividades deportivas y artísticas.

Todas las organizaciones analizadas, a través de programas de cooperación específicos o gracias a las organizaciones de CJ, ofrecen cursos y capacitaciones tanto a nivel de producción, calidad y diversificación, como a nivel de CJ o de producción orgánica. Son prácticas educativas no formales que tienen un fuerte impacto sobre todo cuando están dirigidas a mejorar la productividad de las fincas y a generar más conciencia sobre las posibilidades-capacidades que los mismos pequeños productores poseen para así acceder a los mercados, sin tener que plegarse nuevamente a los intermediarios.

Estas prácticas responden al principio básico de Creación de Capacidades (World Fair Trade Organitation, 2008). Además, el CJ ayuda los miembros de las comunidades a estrechar vinculos, a mantener vivas las redes sociales comunitarias, que dan respuestas alternativas ahí donde las estructuras gubernamentales no llegan. En la Rep. Dominicana, fomenta la integración entre haitianos y dominicanos que trabajan la misma tierra, y también la lealtad y el compromiso de los productores con la organización y la comunidad, hasta el punto que el pequeño productor seguirá vendiendo su producto a la organización, también cuando en momentos muy puntuales el intermediario local esté ofreciendo un mejor precio.

Salud

Otra de las áreas que se benefician directamente de las inversiones sociales del CJ es naturalmente la salud: ahí donde no llegan las autoridades, así como en el caso de la educación, llegan las organizaciones sociales. Vuelvo a mencionar el Informe "Política Social: capacidades y Derechos" de la ODH/PNUD (2010: 302-303, VOL III); algunas de las recomendaciones finales para mejorar el sistema de atención sanitaria en el país son: "Promover y autorizar la multiplicidad de prestadores del primer nivel de atención [...] Modificar los mecanismos de financiamiento del primer nivel de atención, a fin de asegurar que al menos el 30\% de los recursos asignados a la red única de servicios públicos de salud se destine en forma especializada a este nivel de atención [...] Asegurar la implantación de un modelo de atención primaria en el primer nivel, de carácter familiar y comunitario [...] Descentralizar el primer nivel mediante convenios con municipalidades". 
El énfasis que las observaciones y evaluaciones del equipo del PNUD dan al nivel primario, el más descuidado, nos dan una idea de cómo sería importante y estratégico que las autoridades colaboren y proporcionen los justos medios a organizaciones comunitarias, como lo son las organizaciones de pequeños productores, que a nivel local proporcionan respuestas también en el ámbito de la salud. Naturalmente son respuestas sobre todo de nivel primario, aunque no faltan ayudas económicas directas para que socios o miembros de la comunidad puedan acceder a los grandes hospitales y recibir atenciones especiales o se sometan a intervenciones quirúrgicas. CONACADO, por ejemplo, mantiene centros de atención primaria en los cuales trabajan unidades de salud, sin las cuales la atención médica en algunas zonas rurales sería prácticamente imposible.

En Asoarac, garantizan asistencia sanitaria a 160 personas, entre productores, trabajadores y empleados. Además, la asociación organiza operativos médicos, jornadas de vacunación contra varias enfermedades, donaciones de medicinales, entre otras actividades. Banelino destina el $17 \%$ de las inversiones para productores y trabajadores al ámbito de la salud, y un porcentaje similar como inversiones para toda la comunidad. El "Programa de Salud Preventiva" y la construcción del dispensario médico en Mao son dos de las acciones más significativas en este sentido. Coopprobata financia anualmente la construcción de letrinas, un plan básico de atención dental para los productores y sus familias, planes de desparasitación, así como casos especiales de cirugías o estudios especializados.

Estos son solo algunos ejemplos de atención primaria que las organizaciones de productores garantizan a sus socios, sus trabajadores, sus familias y en la medida de lo posible a toda la comunidad. Las siguientes, a mi juicio, son algunas de las líneas de acción de la Propuesta 2010-2030 en las cuales las organizaciones de pequeños productores podrían ser considerados como actores locales protagónicos:

\section{EJE 2: Una sociedad cohesionada, con igualdad de oportunidades y bajos niveles de pobreza y desigualdad} Objetivo Específico Líneas de Acción

Garantizar el acceso a un modelo de atención integral, con calidad y calidez, que privilegie la promoción de la salud y la prevención de la enfermedad, mediante la consolidación del Sistema Nacional de Salud.

\section{Objetivo Específico}

Universalizar el aseguramiento en salud para garantizar el acceso a servicios de salud y reducir el gasto de bolsillo.

1) Impulsar el desarrollo de la red pública de salud y de redes privadas, articuladas por niveles de atención, que brinden atención integral de calidad.

2) Fortalecer los servicios de salud colectiva relacionados con los eventos de cada ciclo de $v$ ida, en colaboración con los gobiernos locales, con énfasis en la prevención de enfermedades transmisibles y el fomento de estilos de vida saludables.

7) Asegurar la provisión efectiva de información a la ciudadanía en torno a su derecho a la salud y a la seguridad social en salud.

Líneas de Acción

1) Fortalecer los mecanismos de afiliación al Sistema de Seguridad S ocial en

Salud, para lograr el aseguramiento universal.

3) Desarrollar, con participación y veeduría ciudadana, un sistema de monitoreo y evaluación de la calidad de los servicios de salud de las prestadoras públicas y privadas.

4) Promover y fortalecer el Seguro contra Riesgos Laborales.

Fuente: MEPYD y CNRE (2010).

\section{Equidad de género}

En la Rep. Dominicana, la tasa de desempleo de las mujeres (26.33\%, promedio entre 2000 y 2008) es casi tres veces mayor que la de los hombres (9.6\%) (MEPYD y CNRE , 2010); entre 2000 y 2007, solo un tercio de las personas ocupadas eran mujeres, y de este tercio solo el 
$25 \%$ residía en zonas rurales; el 32\% de las mujeres están ocupadas en condiciones de subempleo, sobre todo en agricultura y ganadería, donde los subempleados son el $40.34 \%$ de los ocupados; sin embargo, "más de la mitad del empleo adicional promedio total se concentraba en las zonas rurales (37.4 mil) y la ocupación de las mujeres rurales registraba el mayor ritmo de crecimiento (6.5\%)"(PNUD, 2008). A pesar de que la mayoría de los pequeños productores de las organizaciones dominicanas sean hombres, las políticas de género de estas organizaciones están bastante desarrolladas. Sin embargo, hay una diferencia en la división del trabajo que también se refleja a nivel nacional. Cuando se trata de ocupar puestos o cargos administrativos, la presencia de las mujeres es fundamental y sobrepasa de mucho la mitad de los empleados. El caso más paradigmático fue el de Banelino, donde prácticamente toda la administración la llevan las mujeres.

Como del resto en la familia y en la casa de casi todas las familias latinoamericanas. Analizar las acciones de estas organizaciones de productores a favor de una verdadera participación de las mujeres a través de datos cuantitativos es muy complicado. Casos como el de Fundopo, donde el 36\% de los miembros de la Junta Directiva son mujeres pero el porcentaje de productoras sigue siendo el 15\%, o el de Junacas donde solo hay 4 mujeres productoras pero dos de ellas están en la Junta Directiva, son paradigmáticos en este sentido. Sin embargo, el testimonio que Santa Sánchez (productora de CONACADO) dio a Oxfam Wereldwinkels de Bélgica durante la realización de un pequeño documento audiovisual, vale más que muchos discursos bonitos: "Como Usted sabe, antes las mujeres estaban dominadas, pisoteadas, pero ya no [...] principalmente las que pertenecemos a la CONACADO nos hemos liberado mucho [...] sabemos producir el cacao, lo sabemos sembrar, lo sabemos trabajar, lo cortamos, lo picamos y lo llevamos al Bloque; lo mismo que hacen los hombres, lo hacemos las mujeres y eso ha sido a favor de nosotras, de la liberación [...] yo no tenía tanta decisión como la que tengo ahora, antes no me atrevia a decir "voy a coger dinero prestado" [...] pero ya gracias a la liberación a través de la CONACADO, del Bloque, es que sabemos que se va a poder" (OXFAM, 2011).

Aquí, es bueno recordar proyectos como "Café Femenino" ${ }^{4}$, nacido en 2004 y que actualmente involucra a más 1,500 mujeres en seis países: Bolivia, Colombia, Rep. Dominicana, Guatemala, México y Perú, país este último, desde el cual se generó gracias al apoyo directo de la organización comercial estadounidense "OPTCO", Organic Products Trading Company. ${ }^{5}$ Como me decía María Isabel Balbuena de FEDECARES, el Café Femenino "debe de ser procesado, elaborado y manejado por mujeres durante todo el proceso. En realidad las mujeres ya trabajan en la producción pero ni ellas mismas se dan cuenta de lo que están haciendo, porque muchas veces los frutos del trabajo no les llegan directamente y el hombre sigue siendo el que decide como utilizar los beneficios de la venta; pero con este proyecto su café adquiere un valor agregado, y el premio adicional llega directamente a la mujer productora".

\begin{tabular}{|l|l|}
\hline \multicolumn{2}{|c|}{ EJE 2: Una sociedad cohesionada, con igualdad de oportunidades y bajos niveles de pobreza y desigualdad } \\
\hline \multicolumn{1}{|c|}{ Objetivo Específico } & \multicolumn{1}{c}{ Líneas de Acción } \\
\hline \multirow{4}{*}{$\begin{array}{l}\text { Construir una cultura de } \\
\text { igualdad y equidad entren } \\
\text { hombres y mujeres. }\end{array}$} & $\begin{array}{l}\text { 2) Promover una cultura de erradicación de la violencia intrafamiliar y contra la } \\
\text { mujer y fortalecer el sistema de prevención y sanción de la violencia intrafamiliar. }\end{array}$ \\
\cline { 2 - 2 } & $\begin{array}{l}\text { 3) Fomentar la participación pro-activa de la mujer en todos los espacios de la vida } \\
\text { económica, política, social y cultural }\end{array}$ \\
\cline { 2 - 2 } & $\begin{array}{l}\text { 4) Crear mecanismos que faciliten la inserción de la mujer en el mercado } \\
\text { sin discriminación. }\end{array}$ \\
\cline { 2 - 2 } & $\begin{array}{l}\text { 5) Concienciar sobre la igualdad de derechos y la equidad de género y construir } \\
\text { una imagen revalorizada del aporte de la mujer a la economía y la sociedad que } \\
\text { supere los estereotipos tradicionales. }\end{array}$ \\
\hline Fuente: MEPYD y CNRE (2010).
\end{tabular}




\section{Sostenibilidad medioambiental}

En este ámbito, la filosofia del CJ es muy clara: "se incentiva el uso de recursos locales y técnicas de producción medioambientalmente sostenibles y la agricultura ecológica". A través del apoyo a la agricultura sostenible de los pequeños productores, el movimiento por el $\mathrm{CJ}$ impulsa el respeto por el medio ambiente, genera conocimientos medioambientales y favorece toda una cultura que llama a repensar tanto las relaciones entre el ser humano y la naturaleza como los métodos de producción. En un mundo que vive la rápida expansión de los "agronegocios", la agricultura campesina de los pequeños productores rompe el círculo del aumento de gases invernadero (entonces del calentamiento global).

Las prácticas de producción orgánica son asimiladas por todos los grupos de productores que he conocido, aunque los porcentajes de producción orgánica respecto al total varien bastante. La promoción de cultivos bajo sombra, las reforestaciones, la protección de los bosques tropicales y de los ecosistemas forestales nativos son todas prácticas que los pequeños productores latinoamericanos conocen, respetan y reproducen. El utilizo de la prima social para mejorar la sostenibilidad ambiental también es fundamental: la Cooperativa de Polo tiene el apoyo del Ministerio de Medioambiente para reforestar varias pendientes de la zona. Junacas cuenta con un "Equipo técnico en agroecología y medio ambiente" constituido por varios promotores e inspectores internos y lleva adelante un buen trabajo de gestión de residuos sólidos; en febrero de 2010, la organización recibió uno de los premios de "Conservación y Medio Ambiente" de la "Ford Motor Company", para la categoría Conservación y Educación Ambiental y desde 2009 recibe apoyo en temas de biodiversidad por parte del Programa de Pequeños Subsidios (PPS) del PNUD. CONACADO tiene varios programas de cuidado medioambiental, pero aquí me gusta recordar una experiencia del Bloque 2 de Yamasá: para alimentar el fuego de los secadores artificiales, se utilizan las cáscaras de coco que una empresa de San Cristóbal descarta al final del proceso. De esta manera se recicla (cultura casi totalmente ausente en muchos países de América Latina) y no hay que talar nuevos árboles o utilizar gasolina para el combustible. Excelente prueba de que si se dejan trabajar las inteligencias y las capacidades y se escapa de los circuitos convencionales de producción y consumo, se pueden encontrar soluciones baratas y sostenibles.

Asoarac organiza cada año jornadas de recogida de plásticos y basura, instrumentos concretos para difundir una cultura de respeto al medioambiente que las mismas autoridades deberían promover, tanto a nivel nacional como local. La asociación Aprobano mantiene en su estructura organizativa un Consejo de Medio Ambiente y Coopprobata además de recoger basura y desechos plásticos tanto en las fincas como en las comunidades contribuye a las reforestaciones con donaciones anuales de árboles frutales. Todas las organizaciones de pequeños productores de comercio justo reclaman el pago por servicios medioambientales.

Sobre todo los caficultores y cacaocultores en las lomas y montañas del país, contribuyen constantemente al mantenimiento del ecosistema y evitan que los terrenos deslicen hacia los ríos o embalses. En este modo se defiende el recurso natural más importante y estratégico, para toda la sociedad: el agua. Pero, manteniendo los bosques vivos, también se contribuye a la producción de oxígeno reduciendo las concentraciones de carbono. En la "Propuesta de Estrategia Nacional de Desarrollo 2010 - 2030", se habla de "Restaurar y preservar los servicios prestados por los ecosistemas, con énfasis en las cuencas de los ríos, y diseñar e 
instrumentar mecanismos para el pago de servicios ambientales a las comunidades que los protegen". Ahora, no ahorita, es el momento de actuar. El Eje 4 de la Propuesta 2010-2030 está exclusivamente dedicado al manejo medioambiental sostenible y a la adecuación a las consecuencias del cambio climático. Considero que las organizaciones de pequeños productores de $\mathrm{CJ}$ tienen todas las capacidades para constituirse, también en este ámbito, en verdaderos actores de las nuevas políticas medioambientales.

\begin{tabular}{|c|c|}
\hline \multicolumn{2}{|c|}{ EJE 4: Un manejo sostenible del medio ambiente y una adecuada adaptación al cambio cli mático } \\
\hline Objetivo Específico & Líneas de Acción \\
\hline \multirow{5}{*}{$\begin{array}{l}\text { Proteger y aprovechar de } \\
\text { manera sostenible los recursos } \\
\text { naturales y mejorar la calidad } \\
\text { del medio ambiente }\end{array}$} & $\begin{array}{l}\text { 3) Desarrollar sistemas de monitoreo y evaluación del estado del medio } \\
\text { ambiente y los recursos naturales. }\end{array}$ \\
\hline & $\begin{array}{l}\text { 4) Restaurar y preservar los servicios prestados por los ecosistemas, con } \\
\text { énfasis en las cuencas de los ríos, y diseñar e instrumentar mecanismos para } \\
\text { el pago de servicios ambientales a las comunidades que los protegen. }\end{array}$ \\
\hline & $\begin{array}{l}\text { 5) Reforestar los territorios con vocación boscosa con especies endémicas y } \\
\text { nativas. }\end{array}$ \\
\hline & $\begin{array}{l}\text { 7) Apoyar el desarrollo y adopción de tecnologías y prácticas de producción y } \\
\text { consumo ambientalmente sostenibles, desincentivar las contaminantes y } \\
\text { mitigar los daños asociados a actividades altam ente contaminantes. }\end{array}$ \\
\hline & $\begin{array}{l}\text { 9) Promover la educación ambiental y la participación ciudadana en la } \\
\text { valoración, protección y defensa del ambiente y los recursos naturales. }\end{array}$ \\
\hline Objetivo Específico & Líneas de Acción \\
\hline \multirow[t]{2}{*}{$\begin{array}{l}\text { Gestionar el recurso agua de } \\
\text { manera eficiente y sostenible }\end{array}$} & $\begin{array}{l}\text { 2) Planificar de manera coordinada e integral la política hídrica, tomando } \\
\text { como elemento central la cuenca hidrográfica, para la asignación racional y } \\
\text { sostenible de volúmenes de agua para uso humano, ambiental y productivo. }\end{array}$ \\
\hline & $\begin{array}{l}\text { 3) Conservar y gestiona r de manera sostenible los recursos hídricos } \\
\text { superficiales y subterráneos, con el propósito de atenuar los efectos del } \\
\text { cambio climático. }\end{array}$ \\
\hline
\end{tabular}

Esta colaboración con las autoridades, sin embargo, debe pasar por un efectivo reconocimiento por parte del Estado de la necesidad de involucrar a los actores comunitarios y agentes de desarrollo local para darle efectividad a la estrategia nacional de desarrollo y protagonismo a los que cotidianamente contribuyen con su forma de producir y trabajar a un desarrollo humano y sostenible, tanto socialmente como ambientalmente.

\section{Conclusiones}

El Comercio Justo como herramienta de alianza global para un desarrollo humano y sostenible

Los bajos precios en el mercado internacional, la baja productividad, la limitada capacidad de negociación y de acceso al crédito, las deficiencias en la comercialización y distribución de los productos, las pocas infraestructuras para el almacenamiento y el transporte, la escasa protección estatal y la débil capacidad y voluntad institucional para ejecutar, vigilar y evaluar políticas públicas de apoyo al mundo rural, son características que podemos encontrar en muchos países de América Latina. Son unas de las principales barreras que impiden el desarrollo local rural. Eliminar estas barreras y otras privaciones a la libertad de elegir la vida que las personas mejor valoran para su presente y futuro significa, desde el enfoque de Desarrollo Humano propuesto por Amartya Sen, expandir estas libertades y entonces 
permitir el desarrollo desde las mismas necesidades de las personas. En este sentido una estrategia de desarrollo debe llevar a la eliminación de estas privaciones y tener bien claro que una de las principales capacidades humanas es la posibilidad de generar ingresos propios a través de un trabajo digno.

"Ser libres" no significa "poder hacer todo lo que me da las ganas", sino liberarse de las cadenas que aún nos atan, para ejercer todos los derechos humanos fundamentales: ser libre del hambre significa tener el derecho a la alimentación y soberanía alimentaria; ser libre del alfabetismo es tener el derecho a una educación de calidad; ser libre de la explotación, como derecho a un trabajo digno y a un sueldo digno; ser libre de la pobreza, como derecho a condiciones de vida humanas y dignas; ser libre de la esclavitud, como derecho a la libre expresión, etc.

En este sentido, el CJ trabaja sin duda según una perspectiva multidimensional que no deja atrás ninguna de esas libertades-derechos que de verdad corresponden al desarrollo. Su multidimensionalidad es su característica principal. Las estrategias de desarrollo que solo se basan sobre el crecimiento de las exportaciones consideran una sola dimensión. Sin embargo, tampoco en los casos de las relaciones de CJ existen garantías ciertas de que no se cree dependencia de la exportación o de que no se dejen de lado las otras dimensiones. El CJ se inserta en una visión integral del desarrollo en cualquier sociedad. CJ no significa solo comerciar, sino sobre todo repensar éticamente, solidariamente y sosteniblemente la producción, el comercio y el consumo, los tres pilares de la economía mundial. El CJ, entonces, puede ser considerado un importante vehículo para la consecución de una estrategia de desarrollo endógeno, un desarrollo "desde adentro", basado principalmente en los actores locales y comunitarios, verdaderos protagonistas de la construcción de su propio futuro a través de los recursos humanos y naturales locales y el impulso de innovadoras estrategias de producción y consumo.

Cumpliendo con los diferentes criterios del CJ, como pudimos apreciar, los pequeños productores (así como las organizaciones de CJ del Norte) favorecen el desarrollo local en todas sus dimensiones, pero sobre todo amplían el proceso de creación de capacidades que está al centro del paradigma de desarrollo humano ("enfoque de capacidades") de Amartya Sen, y que todas las agencias de desarrollo internacional ya están implementando. Al mismo tiempo, el CJ teje relaciones globales estables y de largo plazo entre los países del "centro" y de la "periferia" y lucha por un cambio de las injustas reglas del comercio internacional también desde arriba, a través de las campañas y del necesario lobby político. Fomenta entonces desde abajo, aquella "Alianza Mundial para el Desarrollo" de la cual se habla en el Objetivo 8, para la cual, sin embargo, no se ha puesta ninguna fecha límite. Es hora de ponerle fecha. La interconexión entre relaciones internacionales y propuestas locales hacen del CJ una de las experiencias "local-globales" de mayor éxito en la búsqueda de alternativas reales al sistema económico actual y sus evidentes carencias. En este sentido, el movimiento del CJ ha ido acercándose siempre más a otros movimientos de "Economía Solidaria", sobre todo en América Latina.

Es justamente desde los países latinoamericanos que más se discuten y se abordan estos temas. El continuo plantearse nuevas perspectivas ha llevado los latinoamericanos a considerar, en palabras del peruano Alfonso Cotera, una perspectiva más "integrada o autocentrada", a través de la cual se entienda que el CJ no es solo "una estrategia de promoción local sustentable, sino además, de [...] generación de empleo, relaciones de equidad entre 
mujeres y hombres, y entre generaciones, y de movilización de valores éticos culturales para el desarrollo desde el espacio local" (ODH-PNUD, 2010). En pocas palabras, una verdadera estrategia local y nacional de desarrollo humano y sostenible.

Faltando muy poco al 2015, es necesario que los organismos y los gobiernos de las Naciones Unidas, las agencias internacionales de cooperación para el desarrollo, las organizaciones no gubernamentales apoyen directamente los esfuerzos de las organizaciones de pequeños productores en todos los países del Mundo, sobre todo los que más dificultades tienen en el logro de los objetivos y donde los efectos del cambio climáticos se están sintiendo de manera más aguda. La multidimensionalidad de la acción de las organizaciones de pequeños productores ligados a los circuitos del Comercio Justo puede garantizar una mejor atención a los diferentes problemas del desarrollo económico, social y políticos de las comunidades rurales y no solamente, y entonces ayudar los esfuerzos hacia el logro de las metas del Milenio. 


\section{Referencias Bibliográficas}

ALAI (2010), "Nuevas tendencias en el agro", Revista América Latina en Movimiento, n. 459, Año XXXIV, II época, Agencia Internacional de Información, Quito, Ecuador, disponible en: [http:/ /alainet.org/publica/459.phtml]

Coscione, M. (2008), El Comercio Justo. Una alianza estratégica para el desarrollo de América Latina, Los Libros de La Catarata, Madrid.

Coscione, M. (se publicará en 2011), Experiencias de Comercio Justo en la República Dominicana: café, cacao y banano, FUNGLODE, Santo Domingo, Rep. Dominicana.

Cotera Fretel, A. (2007), "Comercio Justo: una visión desde América Latina”, en Afirmando prácticas democráticas y estrategias solidarias para un desarrollo sustentable. Memoria del II Encuentro Latinoamericano de Economía Solidaria y Comercio Justo, RIPESS Región Latinoamericana, Nedda Angulo y Massiel Fernández (Compiladoras), Lima: Grupo Red de Economía Solidaria del Perú (GRESP), y Universidad de La Habana, pág. 106, disponible en:

[http://www.ripesslac.net/recursos_galeria/20090223_060941_Memoria_Habana. pdf]

MEPYD y CNRE (2010), "Un Viaje de Transformación hacia un País Mejor. Propuesta de Estrategia Nacional de Desarrollo 2010-2030", Ministerio de Economía, Planificación y Desarrollo, y Consejo Nacional de Reforma del Estado, Santo Domingo, República Dominicana.

ODH-PNUD. (2010). Catedra Fretel: Política social, capacidades y derechos. Recuperado el 2010, de www.repesslac.net:

Http://www.repesslac.net/recursos_galeria/20090223_060941_memoria_habana. pdf

OXFAM. (Marzo de 2011). Conacado- Dominicaanse Republiek. Recuperado el Marzo de 2011, de Oxfam: http://www.oxfamwereldwinkels.be/conacado

PNUD. (2008). Informe de Desarrollo Humano. Republica Dominicana: PNUD.

Murray, D., \& Raynolds, L. T. (2007). Globalization anda its anitionomies. Negotiating a fair trade movement. New York.

World fair trade organization. (2008). principio 7, condiciones laborales. Recuperado el 2008, de http: / / www.wfto.com:

http://www.wfto.com/index.php?option=com_content\&task=view\&id=39\&Itemid= $125 \&$ lang=en

World Fair Trade Organitation. (2008). www.wfto.com. Recuperado el 2008, de Principio 8: http: / / www.wfto.com/index.php?option=com_content\&task=view\&id=39\&Itemid= $125 \&$ lang=en 
Notas

“"Cualquier participación de niños en la producción de productos de Comercio Justo (incluyendo el aprendizaje de un arte tradicional o artesanal) es siempre revelada y supervisada, y no afecta negativamente el bienestar, la seguridad, los requisitos educativos y la necesidad de jugar de los niños". Principio 5, "Trabajo infantil y trabajo forzoso", reconocidos por la "World Fair Trade Organization"; todos los principios se puede consultar entrando en la siguiente página web: http:/ /www.wfto.com/index.php?option=com_content\&task=view\&id=39\&Itemid=125\&lang=en

${ }^{3}$ Todos los testimonios presentes en este trabajo fueron tomados por el autor durante la investigación de campo (junio-julio 2010). La publicación completa, "Experiencias de Comercio Justo en la República Dominicana: café, cacao y banano" se publicará a principios de 2011

${ }^{4}$ Véase: http://www.cafefemenino.com

${ }^{5}$ http://www.optco.com

${ }^{6}$ En el primer capítulo del informe "Política social: capacidades y derechos" de la ODH/PNUD (2010: 3-26, VOL I), se explica claramente el marco teórico del "enfoque de derechos" y del "enfoque de capacidades". 